OPEN ACCESS

Edited by:

Jian-Feng Mao,

Beijing Forestry University, China

Reviewed by:

Detuan Liu

Kunming Institute of Botany, Chinese

Academy of Sciences, China

Yuguo Wang,

Fudan University, China

${ }^{*}$ Correspondence:

Zhe-Chen Q

zqi@zstu.edu.cn

Yu-Kun Wei

ykwei@aliyun.com

Specialty section:

This article was submitted to Evolutionary and Population Genetics,

a section of the journal

Frontiers in Genetics

Received: 30 March 2021

Accepted: 13 April 2021

Published: 11 May 2021

Citation:

Zhou X, Zhang Z-C, Huang Y-B,

Xiao H-W, Wu J-J, Qi Z-C and

Wei Y-K (2021) Conservation

Genomics of Wild Red Sage (Salvia

miltiorrhiza) and Its Endangered

Relatives in China: Population

Structure and Interspecific

Relationships Revealed From 2b-RAD

Data. Front. Genet. 12:688323.

doi: 10.3389/fgene.2021.688323

\section{Conservation Genomics of Wild Red Sage (Salvia miltiorrhiza) and Its Endangered Relatives in China: Population Structure and Interspecific Relationships Revealed From 2b-RAD Data}

\author{
Xuan Zhou',2, Zhi-Cheng Zhang 1,2, Yan-Bo Huang', Han-Wen Xiao'2, Jun-Jie Wu', \\ Zhe-Chen Qi1,3* and Yu-Kun Wei2*
}

1 Zhejiang Province Key Laboratory of Plant Secondary Metabolism and Regulation, College of Life Sciences and Medicine, Zhejiang Sci-Tech University, Hangzhou, China, ${ }^{2}$ Shanghai Key Laboratory of Plant Functional Genomics and Resources and Eastern China Conservation Center for Wild Endangered Plant Resources, Shanghai Chenshan Botanical Garden, Shanghai, China, ${ }^{3}$ Shaoxing Academy of Biomedicine of Zhejiang Sci-Tech University, Shaoxing, China

Red sage (Salvia miltiorrhiza) is a widely used medicinal plant for treatment of cardiovascular and cerebrovascular diseases. Because of excessive excavation by huge market demand and habitat loss by human activities, the wild population resources of S. miltiorrhiza have reduced drastically in recent years. Meanwhile, population status of two closely related species $S$. bowleyana and $S$. paramiltiorrhiza were in a trend of decreasing due to their potential replacement of S. miltiorrhiza. Particularly, S. paramiltiorrhiza was threatened and endemic to a small region in eastern China. However, to date there has been no conservation genetic research reported for wild S. miltiorrhiza population and its endangered relatives. Assess the wild germplasm diversity for $S$. miltiorrhiza and its related species would provide fundamental genetic background for cultivation and molecular breeding of this medicinally important species. In the present study, we investigated the genetic diversity, population structure, and intra/inter-specific differentiation of $S$. miltiorrhiza and above two relatives using $2 b-R A D$ genome-wide genotyping method. By investigating 81 individuals of S. miltiorrhiza, 55 individuals of $S$. bowleyana and 15 individuals of $S$. paramiltiorrhiza from 23 locations in China, we obtained 23,928 SNPs in total. A comparatively high genetic diversity was observed in S. miltiorrhiza ( $\pi=0.0788, H_{e}=0.0783 \pm 0.0007$ ). The observed and expected heterozygosity in populations of these three species ranged from 0.0297 to 0.1481 and 0.0251 to 0.831 , respectively. Two major lineage groups were detected in the examined S. miltiorrhiza populations. The results indicated that Dabie Mountain as a genetic diversity center of $S$. miltiorrhiza and possible complex inter-specific genetic 
exchange/hybridization occurred between S. miltiorrhiza and the two relatives. We suggest that strategic conservation and germplasm preservation should be considered not only for wild populations of $S$. miltiorrhiza, but also for its related S. bowleyana and S. paramiltiorrhiza.

Keywords: Salvia miltiorrhiza, 2b-RAD, population genomics, conservation, genetic diversity, Salvia bowleyana, Salvia paramiltiorrhiza, wild germplasm

\section{INTRODUCTION}

Medicinal plants are invaluable resource of maintaining health for the majority of world's population. At present, more than $80 \%$ of the world's population depends on herbal medicine for primary healthcare needs, while millions gain income from their wild harvest or cultivation, or are involved in their trading or processing (Hamilton, 2004). With the growing human populations, the demand for medicinal plants kept growing rapidly throughout the world for the past decades for their usage in herbal drugs and natural health products (Bentley, 2010; Jamshidi-Kia et al., 2018). According to IUCN, 50,000 and 80,000 flowering plant species are estimated to be used for pharmaceutical purposes around the world. For instance, China and India have the highest numbers of medicinal plants used, with 11,146 and 7,500 species, respectively. Among these numbers, 90\% species were directly harvested from wild resources (Sarwat et al., 2011; Chen et al., 2016). Overexploitation and habitat destruction by human activity has led to an alarming number of medicinal plant species under threat of extinction. About 15,000 species are exposed to a risk of extinction due to high harvesting and destruction of habitats. A high proportion of medicinal plant species have become endangered in some species rich areas, threatening the regional biodiversity and ecosystem stability, especially in developing countries (Heywood and Iriondo, 2003; Hamilton, 2008; Bentley, 2010; Jamshidi-Kia et al., 2018).

One way to relief this situation is domestic cultivation. A number of governments and agencies are recommending that wild medicinal species should be brought into cultivation systems (WHO et al., 1993). Increased cultivation of medicinal plant is not only a means for meeting the huge market demands for drug and herbal remedies based on medicinal plant, but also a way to decrease harvest volume on wild populations, benefiting to the recovery of their wild resources (Schippmann et al., 2002; Canter et al., 2005). However, medicinal plant production through cultivation would probably result in genetic diversity reduce of cultivars due to founder effect and artificial selection, resembling the genetic bottleneck during the domestication of cereal species (Doebley et al., 2006). Decreased genetic diversity will lead them susceptible to pests, diseases, abiotic stress. Meanwhile, cultivation could also negatively affect the incentives to conserve wild populations (Schippmann et al., 2006). Wild population and relatives with genetic resources have proven to be very valuable in agricultural breeding practice (Tyack et al., 2020). The conservation of wild populations could maintain genetic diversity and allows for the continuing evolution of the gene pool as a germplasm resource for future crop improvement (Tyack et al., 2020; Vining et al., 2020). In this regard, systematic collection and analyses of genetic diversity and relationships in wild medicinal plant populations can lay important foundations for the conservation of species as well as designing strategies for development of commercially relevant varieties (Govindaraj et al., 2015).

Salvia miltiorrhiza Bunge (Lamiaceae), the red sage, is an indigenous medicinal herb in China. Wild populations of S. miltiorrhiza usually grows on the hillsides, stream banks and understories in broad-leaved deciduous forests in central and eastern China. Its dried roots, commonly known as Danshen, have long been used in traditional Chinese medicine for treatment of cardiovascular and cerebrovascular diseases (Wang, 2010; Zhang et al., 2014). The production of S. miltiorrhiza in China achieved a total revenue of around 75 million USD in 2015 (Kum, 2019). Due to the huge market demand, although mostly harvested from cultivation, the wild populations of $S$. miltiorrhiza reduced drastically in recent years by excessive excavation and habitat destruction (Yang et al., 2017). Moreover, germplasm depression was frequently observed in cultivated S. miltiorrhiza. Meanwhile, two closely related species S. bowleyana Dunn and S. paramiltiorrhiza Li and Huang are also under a pressure of population decrease as frequent substitutes to S. miltiorrhiza (Field observation in the past decade). Particularly, populations of S. paramiltiorrhiza was critically threatened and can only be found in restricted areas in Hubei and Anhui, China (Huang and Li, 1981; Hao et al., 2015). Morphologically, these three species can be distinguished from each other mainly by their flower and root colors (Figure 1). However, previous phylogenetic studies based on plastid DNA and a few nuclear loci showed that their evolutionary relationships remain unresolved (Li et al., 2013; Hu et al., 2018). As wild populations and relatives as germplasm resources may provide infinite potentials for superior breeding varieties to improve the quality of cultivars (Vining et al., 2020; Wang et al., 2020), it is essential to conserve and assess the genetic diversity of $S$. miltiorrhiza and its relatives in natural populations.

Molecular markers are playing a significant role in studying population genetics of medicinal plants (Sarwat et al., 2011; Wang et al., 2020). With suitable genetic makers, we can understand the germplasm diversity, such as single nucleotide polymorphism (SNP), allele frequencies, extend and distribution of genetic diversity, and population structure, under a rigorous mathematical framework. Such information gained can be then referred to devise a proper conservation strategy and genebank management. With its ultra-high-throughput, nextgeneration sequencing (NGS) technology could provide an 


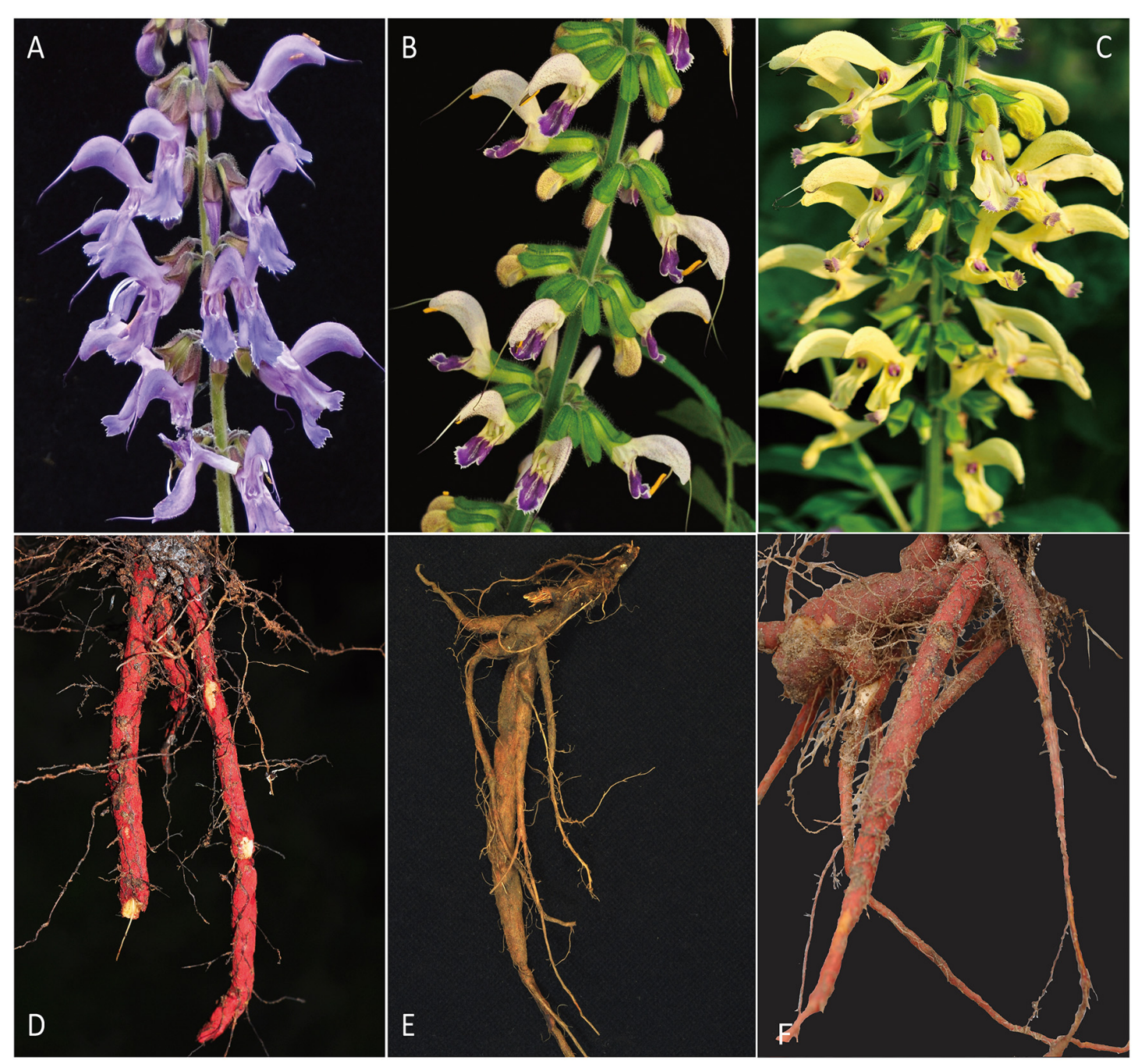

FIGURE 1 | Flower and root morphology the three studied species. (A) flower of S. miltiorrhiza. (B) flower of S. bowleyana. (C) flower of S. paramiltiorrhiza. (D) root of S. miltiorrhiza. (E) root of $S$. bowleyana. (F) root of S. paramiltiorrhiza.

efficient toolkit in producing large amount of SNP markers for organisms through simplified genome sequencing, for example, restriction site-associated DNA sequencing (RADSeq), genotyping-by-sequencing (GBS) or other related techniques (Davey et al., 2011; Peterson et al., 2012; Andrews et al., 2016). 2b-RAD, characterized by producing uniform fragments generated by type IIB restriction endonuclease, is a flexible and cost-effective method outperform other RAD-seq methods with higher markers numbers, repeatability, and genomic coverage density (Wang et al., 2012).

In present study, we assessed the genetic diversity of S. miltiorrhiza and two closely related species (S. bowleyana and $S$. paramiltiorrhiza) using $2 \mathrm{~b}-\mathrm{RAD}$ sequencing. We investigated the phylogenetic relationships and several aspects of the population genetics of focusing on wild S. miltiorrhiza populations including genetic diversity, population structure, and differentiation, as well as estimation of demographic history. We hope the methods and results presented here could be useful in guidance of wild germplasm conservation and management for S. miltiorrhiza and other medicinal plants in the future.

\section{MATERIALS AND METHODS}

\section{Sampling and DNA Extraction}

A total of 151 individuals from 23 sites in central and eastern China were sampled and analyzed in this study, including S. miltiorrhiza (81 individuals from 13 wild populations), S. bowleyana (55 individuals from 9 wild populations) and S. paramiltiorrhiza (15 individuals from 1 wild populations) (Supplementary Table 1). Our sampling focused on an area where populations of all three species occur, in order to assess their potential gene flows. Leaves collected from each individual were dried in silica gel. Genomic DNA was extracted from dried leaf powder using a modified CTAB method (Allen et al., 2006). First, gel-dried leaf samples were put into liquid nitrogen and 
quickly ground to powder; the powder was then immediately transferred into a clean $1.5 \mathrm{ml}$ microcentrifuge tube and mixed immediately with $600 \mu \mathrm{l} 2 \times \mathrm{CTAB}$ extracting solution and incubated in a water bath at $65^{\circ} \mathrm{C}$ for $45 \mathrm{~min}$. The samples were then centrifuged twice at $12,000 \mathrm{rpm}$ for $10 \mathrm{~min}$, after which the supernatant was transferred to another clean $1.5 \mathrm{ml}$ microcentrifuge tube. Then $5 \mu \mathrm{l}$ DNase free RNase $(10 \mu \mathrm{g} / \mu \mathrm{l})$ was added and incubate at $37^{\circ} \mathrm{C}$ for $15 \mathrm{~min}$, intermittently mixed up for 2-3 times. The same volume of chloroform-isoamyl alcohol (24:1) was then added and mixed up the phases by gentle inversion. The sample was centrifuged at $12,000 \mathrm{rpm}$ for $5 \mathrm{~min}$, after which the supernatant was transferred to a new $1.5 \mathrm{ml}$ centrifuge tube and mixed with the same volume of isopropanol, then incubated at $-20^{\circ} \mathrm{C}$ for about $1 \mathrm{~h}$. The solution was centrifuged at $12,000 \mathrm{rpm}$ for $10 \mathrm{~min}$, then the supernatant liquor was carefully discarded. The DNA was then cleaned twice with $75 \%$ ethanol and air dried at room temperature. The cleaned DNA was then dissolved in $50 \mu \mathrm{l}$ TE. DNA quantity was assessed with 1\% agarose gel electrophoresis, and Qubit 3.0 fluorescent quantitative assay and NanoVue were used to test purity and density.

\section{Multi-IsoRAD Sequencing and SNP Calling}

Library construction and sequencing were conducted following Wang et al. (2016), an updated 2b-RAD approach that allows the preparation of five concatenated isoRAD tags for Illumina pairedend sequencing. After DNA extraction and quality control, the high-quality genomic DNA (600 ng) was digested with restriction enzyme of IIB type (BsaXI) at $37^{\circ}$ for $1 \mathrm{~h}$, followed by enzyme heat inactivation at $65^{\circ}$ for $20 \mathrm{~min}$. The ligation reaction was performed by combining $5 \mu \mathrm{l}$ of digested DNA with $20 \mu \mathrm{l}$ of a ligation master mix containing $0.4 \mu \mathrm{M}$ each of library-specific adaptors with fully degenerate cohesive ends ( $\left.5^{\prime}-\mathrm{NNN}-3^{\prime}\right)$, $0.2 \mathrm{mM}$ adenosine $5^{\prime}$-triphosphate (New England Biolabs), and 1000 U T4 DNA ligase. Then PCR amplification was performed. Ligation was carried out at $16^{\circ}$ for $3 \mathrm{~h}$, with subsequent heat inactivation for $10 \mathrm{~min}$ at $65^{\circ}$. In Multi-isoRAD protocol, the single-tag constructs can be further digested by SapI to generate distinct cohesive ends and then ligated in a predefined order to produce five concatenated tags for Illumina paired-end sequencing using modified adaptors and biotin-labeled primers. After individual libraries were pooled into equimolar amounts, the pool quality was verified on Agilent 2100 Bioanalyzer. The paired-end RAD-seq were generated using Illumina Hiseq $\mathrm{X}$ ten platform (Illumina, San Diego, CA, United States) using 150 bp pair-end sequencing.

Quality control were performed with Trimmomatic (Bolger et al., 2014) according to the following criteria: (a) any reads with adapter; (b) any reads with $>50 \%$ bases having phred quality $<15$; (c) any reads with $>8 \%$ unidentified nucleotides (N). After that, the high-quality reads of each sample were aligned to a reference genome of S. miltiorrhiza (Xu et al., 2016) using the SOAP2 (version 2.21) program following the protocols by Li et al. (2009). A maximum of two mismatches (-v 2) was allowed for each read, and those that mapped onto more than one position in the genomic reference sequence were excluded $(-\mathrm{r} 0)$. Reads with depths $<3$ were not counted. The match mode was set to "find the best hits" (-M 4). and the SNPs were obtained based on a maximum likelihood algorithm and filtered by the RADtyping program (Fu et al., 2013). Finally, Quality control was performed using VCF tools (Danecek et al., 2011) to remove SNPs showing a call rate of less than 90\%, a minor allele frequency (MAF) of less than 0.05 or significant deviation from Hardy-Weinberg equilibrium $(p<0.05)$.

\section{Phylogenetic Reconstruction}

To infer relationships among samples, we performed phylogenetic analyses with concatenated SNP dataset using maximum likelihood (ML) method. The optimal substitution models for the ML phylogenetic analyses was determined by ModelFinder program (Kalyaanamoorthy et al., 2017) using the Bayesian information criterion (BIC), as implemented in IQ-TREE v2 (Minh et al., 2020). The ML analysis was conducted with IQ-TREE. Five searches were compared to ensure identical topologies. Statistical supports were analyzed using 10,000 replicates of ultrafast bootstrapping (UFBoot: Minh et al., 2013) and 10,000 bootstrap replicates of the Shimodaira/Hasegawa approximate likelihood ratio test (SH-aLRT: Guindon et al., 2010).

\section{Analyses of Genetic Diversity and Structure}

Population genetic statistics, including observed and expected heterozygosity $\left(H_{e}, H_{\mathrm{o}}\right)$, nucleotide diversity $(\pi)$, and Wright's F statistics of genetic differentiation $\left(F_{s t}\right)$ and inbreeding coefficient $\left(F_{I S}\right)$ were assessed using the "populations" program in Stacks v2.2 (Rochette et al., 2019), and the pairwise statistics between populations were performed using non-parametric tests. Principal component analysis (PCA) and population structure analysis with all SNPs were performed using Plink v1.9 (Purcell et al., 2007) and ADMIXTURE v1.3.0 (Alexander et al., 2009), respectively. Population genetic variation was calculated using an analysis of molecular variance (AMOVA) with packages adgenet v2.1 (Jombart and Ahmed, 2011) and poppr v2.9 (Kamvar et al., 2015) in R v4.0.3 (R Development Core Team, 2018). Pairwise comparisons of $F_{s t}$ among and within three studied species were estimated. To investigate the correlation between genetic distance and geographic distances, Mantel test was performed using GenAlEx v6.5 (Peakall and Smouse, 2012). Geographic distance between field sampling sites were calculated using geosphere v1.5 package, and genetic distances were obtained with nei.dist function of poppr package.

\section{Demographic History and Gene Flow}

Recent demographic dynamics of S. miltiorrhiza was investigated following Liu et al. (2020). A python script easySFS ${ }^{1}$ was used to generate the folded site frequency spectrum (SFS) formatted file with VCF files and sample list file, and demographic history was then inferred using the program Stairway plot v.0.2

\footnotetext{
${ }^{1}$ https://github.com/isaacovercast/easySFS
} 
(Liu and $\mathrm{Fu}, 2015$ ). The mutation rate of $S$. miltiorrhiza was set to $2.67 \times 10^{-8}$ per site per generation (unpublished data, based on genomic scale mutation and divergence between $S$. miltiorrhiza and $S$. bowleyana). The generation time was set to 1 year per generation. The program BAYESASS version 3.0.3 (Wilson and Rannala, 2003) was used to examine contemporary gene flow (over the last few generations) and migration rates. Analyses were performed inter-specific and inter-cluster levels separately. We conducted five independent runs using $10^{7}$ iterations, a burn-in length of $10^{6}$ and a sampling interval of $10^{3}$ steps. To reach the recommended acceptance rates, Markov chain Monte Carlo mixing parameter values for migration rates (gene flow), allele frequencies, and inbreeding coefficients were adjusted to $0.50,0.95$, and 0.50 , respectively. To confirm convergence, each run was executed with different seed numbers, and trace files were examined for consistent oscillations using Tracer 1.7 (Rambaut et al., 2018).

\section{RESULTS}

\section{Summary Statistics}

A total of 1,097,351,870 raw reads were generated from 151 individuals. After all quality filter, we retained a total of $941,122,629$ reads from the initial raw reads, with an average of $6,232,600$ reads per sample. The average number of unique tags was 50,031 after removing the unique tags whose sequencing depth was less than 3. Mean sequence depths across all samples was $32 \times$, ranging from $17.77 \times$ to $58.41 \times$. After filtering, we obtained 23,928 SNPs in all samples for further analyses.

\section{Phylogenetic Relationships}

The ML phylogeny inferred from TVM + F + R2 model identified four well-supported clades (A-D), with SHaLRT/UFBoot values displayed above branches (Figure 2). Except several subclades in clade $\mathrm{C}$, all other nodes received robust statistical supports. Clade A includes individuals from 7 populations of $S$. miltiorrhiza and one population of S. paramiltiorrhiza. Salvia paramiltiorrhiza population is well-embedded in S. miltiorrhiza, forming a monophyly with population SMHX. Clade B includes individuals from a single S. bowleyana population (SBZL) of south Zhejiang province. In clade $\mathrm{C}, \mathrm{SBAQ}$ and one SBAH individual of $S$. bowleyana formed the basal clade C1 (SH-aLRT/UFBoot: 99.6/87). Clade $\mathrm{C} 2$ (SBHL and SBHY) is sister to clade C3 + C4. Clade C3 includes SBAH and SBZS of S. bowleyana populations is in turn sister to three S. miltiorrhiza populations (SMAT, SMAH and SMAX) with weak support (SH-aLRT/UFBoot: 32.4/54). SMHS, SMAJ and SMHB of S. miltiorrhiza and SBAY, SBZT and SBJY of S. bowleyana showed inter-mixed relationships in clade D.

\section{Genetic Diversity and Population Structure}

In S. miltiorrhiza populations, the observed and expected heterozygosity range from 0.0297 to 0.0647 and 0.0251 to 0.0571 , respectively. The mean values of $\pi$ and $F_{I S}$ are 0.0282 to 0.0641 and -0.0066 to 0.0095 , respectively. Significant difference of the genetic parameters were detected among the populations $\left(H_{0}\right.$ : $\left.p=0.000 ; H_{e}: p=0.000 ; \pi: p=0.000 ; F_{I S}: p=0.000\right)$. In $S$. bowleyana populations, $H_{o}$ and $H_{e}$ range from 0.0353 to 0.1481 and 0.0322 to 0.0831 , respectively. The mean values of $\pi$ and $F_{I S}$ are 0.0371 to 0.0890 and -0.1129 to 0.0078 . Except $F_{I S}$, significant difference of the genetic parameters were also detected among the $S$. bowleyana populations $\left(H_{o}: p=0.000 ; H_{e}: p=0.000\right.$; $\left.\pi: p=0.000 ; F_{I S}: p=0.028\right)$. The mean values of $H_{o}, H_{e}, \pi$ and $F_{I S}$ in $S$. paramiltiorrhiza are $0.0464,0.0445,0.0462$, and 0.0018 , respectively. High values of private alleles are observed in some populations sampled in the present study (Table 1). Average private alleles in a population are 239 (S. miltiorrhiza, highest $=995$ in SMHB), 787 (S. bowleyana, highest $=4790$ in SBZT) and 353 (S. paramiltiorrhiza), respectively. The summary statistics of genetic diversity of all three species are given in Table 1.

In the Admixture analysis, the $\mathrm{CV}$ errors continued to decrease as $\mathrm{K}$ increased in value, giving no clear indication of the appropriate $\mathrm{K}$ for the study populations. As such, conclusions on population substructuring were based on visual inspection of the admixture plots (Figure 3) and PCA plots (Figure 4). A graphic representation of cluster structure analysis is depicted in Figure 3 with $\mathrm{K}$ from 3 to 5 . The species and the genotype boundary between groups is clearer at $K=5$. Salvia paramiltiorrhiza is dominated by cluster 1 . Four major clusters were observed in S. miltiorrhiza (cluster 2-5). It also indicated that cluster 4 and 5 were shared by six populations of $S$. miltiorrhiza (SMAX, SMAH, SMAT, SMAJ, SMHS, and SMHB) and S. bowleyana (Figure 3, $K=5$ ). Different degrees of inter/intra-specific admixtures were observed in the studied populations. Further insights into population structure were obtained by plotting the five genetic clusters on the map (Figure 5). Geographical distribution of the genetic clusters at population level did not show conspicuous genetic structure.

For PCA plots, PC1, PC2, and PC3 explained approximately $33.43 \%$ of the total variation. When we grouped the samples according to the five clusters in Admixture analysis, cluster 1 and 2 overlapped to each other in PC1-PC2 plot (Figure 4A), while showed a distant relationship in PC1-PC3 plot (Figure 4B). Cluster 3, a genetic group from S. miltiorrhiza showed close relationship with cluster 4 in both plots. In cluster 4 , individuals of $S$. miltiorrhiza and $S$. bowleyana mixed together (Figures 4A,B). Cluster 5 showed isolated relationships with other clusters. It is also noted that while share the same cluster 5, individuals of S. miltiorrhiza (SMHB) show a clear distance with S. bowleyana populations (SBZT and SBJY).

AMOVA analyses was performed among species and within each species separately. For within-species analysis, we grouped the populations according to the five ancestry clusters recovered by Admixture analysis. The results revealed that inter-specific differences account for $13.12 \%$ of the genetic variation. Most of genetic variation is due to the differences within populations $(49.78 \%)$ (Table 2). The same pattern was observed in S. miltiorrhiza analyses, that high percentage of the genetic variance $(65.52 \%)$ came from the differences within populations. In $S$. bowleyana, most of the genetic variation $(56.36 \%)$ is due to 


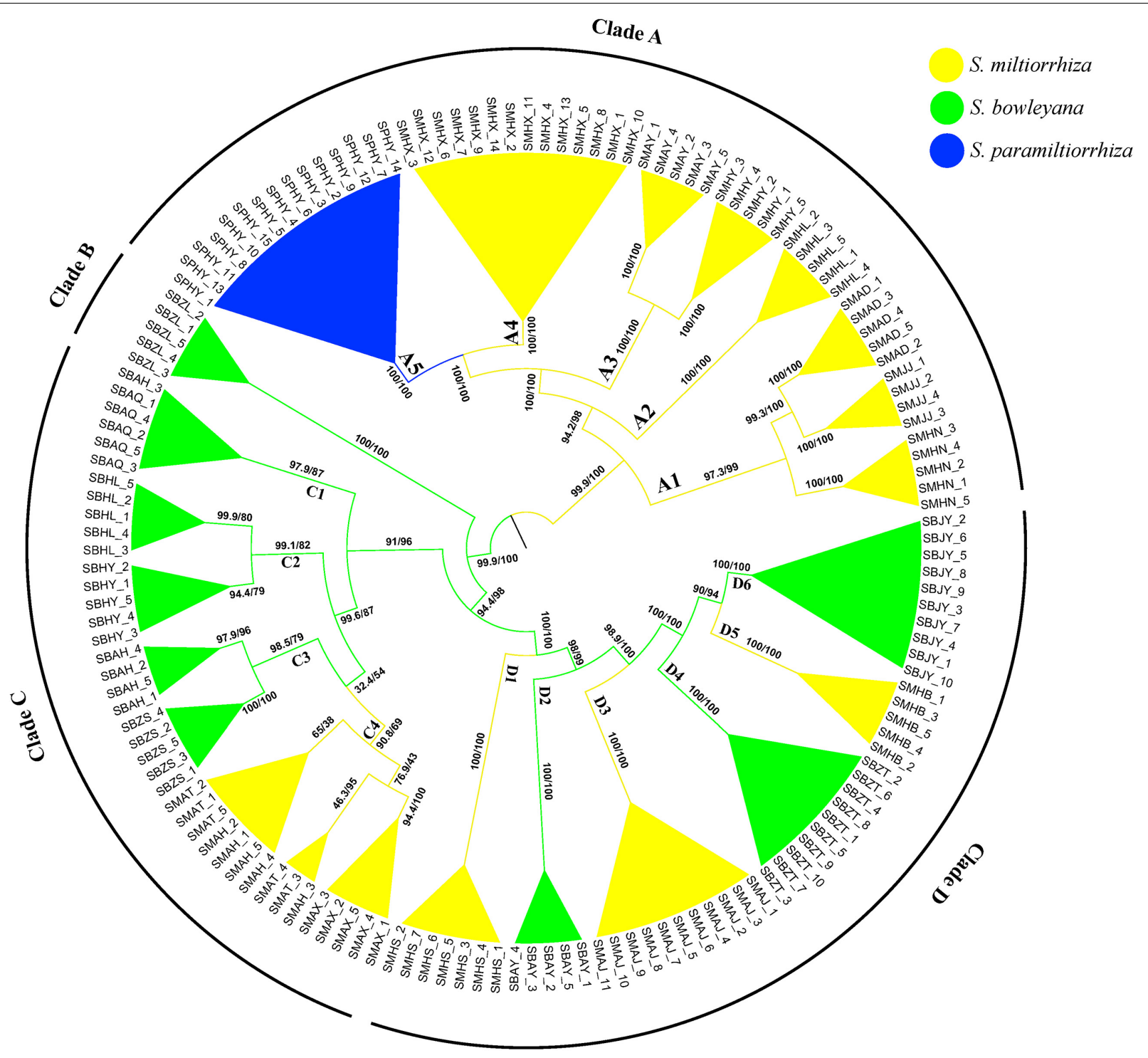

FIGURE 2 | Phylogenetic relationship between S. miltiorrhiza, S. bowleyana, and S. paramiltiorrhiza sampled populations using Maximum Likelihood methods based on SNPs. Bootstrap supports (SH-aLRT/UFBoot values) are indicated at each node. The whole tree can be divided into four major clade (Clade A, B, C, D). Subclade for each species are marked by different color, and the tips label are the individuals name.

differences between populations within clusters. Pairwise genetic differentiation $\left(F_{s t}\right)$ between samples from sampling sites were different significantly, as shown in Supplementary Table 2, and the results are consistent with the genetic variation between populations by AMOVA. Mantel test failed to detect a significant isolation by distance (IBD) based on the geographical distance and genetic divergence matrices in both species (S. miltiorrhiza: $R^{2}=0.0013, p=0.460 ; S$. bowleyana: $\left.R^{2}=0.0174, p=0.450\right)$.

\section{Migration Rates and Gene Flow Analysis}

For inter-specific migrations, we detected significant gene flow from S. bowleyana to S. miltiorrhiza (21.45\%) estimated in BAYESASS (Table 3). It is consistent with the Admixture results that several S. miltiorrhiza populations shared clusters with
S. bowleyana. The second highest but not significant migration was inferred from S. paramiltiorrhiza to S. miltiorrhiza. For intercluster runs, overall low levels of contemporary gene flow were revealed. The highest migration rates were inferred from the cluster 5 to $4(6.44 \%)$, cluster 1 to $2(3.23 \%)$, and cluster 3 to 4 (2.56\%). Cluster 4 received comparatively higher gene flows from other clusters. This is also observed in Admixture analyses that most individuals from cluster 4 showed differential admixture levels with other clusters.

\section{Effective Population Size and Demographic History}

Effective population size and demographic history of three studied species were inferred based on SNP frequency spectra 
TABLE 1 | Population genetic statistics of S. miltiorrhiza, S. bowleyana, and S. paramiltiorrhiza in this study: observed heterozygosity $\left(H_{0}\right)$, expected heterozygosity $\left(H_{e}\right)$, genetic diversity $(\pi)$, inbreeding coefficients $\left(F_{I S}\right)$.

\begin{tabular}{|c|c|c|c|c|c|c|}
\hline Taxon & Pop ID & Private alleles & $H_{o}$ & $H_{e}$ & $\pi$ & $F_{I S}$ \\
\hline \multirow[t]{9}{*}{ S. bowleyana } & SBAH & 16 & $0.0591 \pm 0.0010$ & $0.0547 \pm 0.0009$ & $0.0614 \pm 0.0010$ & $0.0058 \pm 0.0029$ \\
\hline & SBAQ & 141 & $0.0353 \pm 0.0009$ & $0.0322 \pm 0.0007$ & $0.0371 \pm 0.0008$ & $0.0041 \pm 0.0048$ \\
\hline & SBAY & 146 & $0.0484 \pm 0.0010$ & $0.0416 \pm 0.0008$ & $0.0471 \pm 0.0009$ & $-0.0018 \pm 0.0041$ \\
\hline & SBHL & 13 & $0.0581 \pm 0.0010$ & $0.0545 \pm 0.0008$ & $0.0612 \pm 0.0010$ & $0.0073 \pm 0.0030$ \\
\hline & SBHY & 11 & $0.0614 \pm 0.0011$ & $0.0552 \pm 0.0009$ & $0.0619 \pm 0.0010$ & $0.0020 \pm 0.0025$ \\
\hline & SBJY & 1918 & $0.0508 \pm 0.0012$ & $0.0405 \pm 0.0009$ & $0.0430 \pm 0.0009$ & $-0.0142 \pm 0.0105$ \\
\hline & SBZL & 29 & $0.0553 \pm 0.0010$ & $0.0521 \pm 0.0008$ & $0.0587 \pm 0.0010$ & $0.0077 \pm 0.0035$ \\
\hline & SBZS & 22 & $0.0551 \pm 0.0010$ & $0.0519 \pm 0.0009$ & $0.0587 \pm 0.0010$ & $0.0078 \pm 0.0035$ \\
\hline & SBZT & 4790 & $0.1481 \pm 0.0027$ & $0.0831 \pm 0.0015$ & $0.0890 \pm 0.0016$ & $-0.1129 \pm 0.0154$ \\
\hline \multirow[t]{13}{*}{ S. miltiorrhiza } & SMAD & 97 & $0.0408 \pm 0.0009$ & $0.0398 \pm 0.0008$ & $0.0449 \pm 0.0009$ & $0.0095 \pm 0.0039$ \\
\hline & SMAH & 8 & $0.0489 \pm 0.0010$ & $0.0452 \pm 0.0008$ & $0.0526 \pm 0.0009$ & $0.0083 \pm 0.0054$ \\
\hline & SMAJ & 516 & $0.0563 \pm 0.0010$ & $0.0535 \pm 0.0009$ & $0.0566 \pm 0.0009$ & $0.0025 \pm 0.0085$ \\
\hline & SMAT & 12 & $0.0647 \pm 0.0011$ & $0.0571 \pm 0.0009$ & $0.0641 \pm 0.0010$ & $-0.0003 \pm 0.0028$ \\
\hline & SMAX & 39 & $0.0548 \pm 0.0010$ & $0.0493 \pm 0.0008$ & $0.0560 \pm 0.0010$ & $0.0031 \pm 0.0039$ \\
\hline & SMAY & 55 & $0.0469 \pm 0.0010$ & $0.0408 \pm 0.0008$ & $0.0462 \pm 0.0009$ & $-0.0009 \pm 0.0036$ \\
\hline & SMHB & 995 & $0.0456 \pm 0.0011$ & $0.0401 \pm 0.0008$ & $0.0452 \pm 0.0009$ & $-0.0002 \pm 0.0053$ \\
\hline & SMHL & 185 & $0.0297 \pm 0.0009$ & $0.0251 \pm 0.0007$ & $0.0282 \pm 0.0007$ & $-0.0022 \pm 0.0041$ \\
\hline & SMHN & 77 & $0.0520 \pm 0.0011$ & $0.0450 \pm 0.0008$ & $0.0505 \pm 0.0009$ & $-0.0027 \pm 0.0030$ \\
\hline & SMHS & 500 & $0.0430 \pm 0.0010$ & $0.0373 \pm 0.0008$ & $0.0407 \pm 0.0009$ & $-0.0041 \pm 0.0054$ \\
\hline & SMHX & 298 & $0.0443 \pm 0.0009$ & $0.0425 \pm 0.0008$ & $0.0443 \pm 0.0008$ & $0.0019 \pm 0.0090$ \\
\hline & SMHY & 130 & $0.0378 \pm 0.0010$ & $0.0321 \pm 0.0007$ & $0.0362 \pm 0.0008$ & $-0.0022 \pm 0.0038$ \\
\hline & SMJJ & 195 & $0.0370 \pm 0.0010$ & $0.0284 \pm 0.0007$ & $0.0332 \pm 0.0008$ & $-0.0066 \pm 0.0030$ \\
\hline S. paramiltiorrhiza & SPHY & 353 & $0.0464 \pm 0.0009$ & $0.0445 \pm 0.0008$ & $0.0462 \pm 0.0008$ & $0.0018 \pm 0.0095$ \\
\hline
\end{tabular}

and displayed on stairway plots (Figure 6). For S. miltiorrhiza, an initial shallow population contraction occurred at ca. 60 kya (thousand years ago), after which followed a population expansion and then a flat period until 8 kya. Then, a second more severe population bottleneck occurred during ca. 8-6 kya, which followed another population expansion and then a flat growth period maintained to present. For S. bowleyana populations, a deep population bottleneck was also detected around 8-6 kya, which followed a similar population dynamics as S. miltiorrhiza. Salvia paramiltiorrhiza experienced an early population contraction at ca. $104 \mathrm{kya}$, and then rapidly expanded and maintained its population size to present.

\section{DISCUSSION}

Preserving the biological and genetic diversity from wild plant species were considered as an essential and effective way of conservation and sustainable use of medicinal plants (Hamilton, 2008; Chen et al., 2016; Vining et al., 2020). Therefore, it is important to characterize genetic diversity and population structure of targeted species and its relatives, especially threatened ones (Lee et al., 2018, 2020). The genetic diversity in three studied species are relatively high compare to other medicinal herbs, for example, Coptis species (Wang et al., 2020), Valeriana officinalis (Boczkowska et al., 2020), Aconitum austrokoreense (Lee et al., 2018). Especially for S. paramiltiorrhiza, characterized by yellow flower and longer tube without hair annulus, high genetic diversity was observed (0.0462) despite its small population size and restricted distribution. This could be due to its outcrossing behavior and possible historical introgression with S. miltiorrhiza, which is supported by PCA plot (Figure 4A). Although phylogenetic analysis indicated that $S$. paramiltiorrhiza is nested within S. miltiorrhiza populations (Figure 2), Admixture analysis (Figure 3, $K=5$ ) and PCA plot (Figure 4B) revealed that it's a distinct cluster to $S$. miltiorrhiza and $S$. bowleyana populations. Pairwise $F_{s t}$ also indicates that SPHY, the S. paramiltiorrhiza population, is significantly differentiated from all other studied populations of S. miltiorrhiza and S. bowleyana (Supplementary Table 2). Geographically, S. paramiltiorrhiza is isolated from other sampled populations (Figure 5). However, admixture from S. paramiltiorrhiza to $S$. miltiorrhiza were detected in population SMHX, SMAY, and SMAD. A more extensive field examination is needed to confirm whether coexistence of S. miltiorrhiza is occurred in its distribution range.

Relationships between $S$. miltiorrhiza and $S$. bowleyana indicated a possible complex history. Morphologically, S. bowleyana can be distinguished from S. miltiorrhiza by its brownish root and yellow to pink upper corolla lips (Figure 1). Genetic analyses indicated that six populations (SMAX, SMAH, SMAT, SMAJ, SMHS, SMHB) of S. miltiorrhiza shared cluster 4 and 5 with $S$. bowleyana populations in Admixture analysis, which is supported by phylogenetic analysis (clade $\mathrm{C}$ and D, Figure 2). All of these six populations distributed in the Dabie Mountain area, which overlaps the northern distribution range of S. bowleyana (Figure 5). On one hand, we assumed that the frequent gene flows and possible hybridization might 


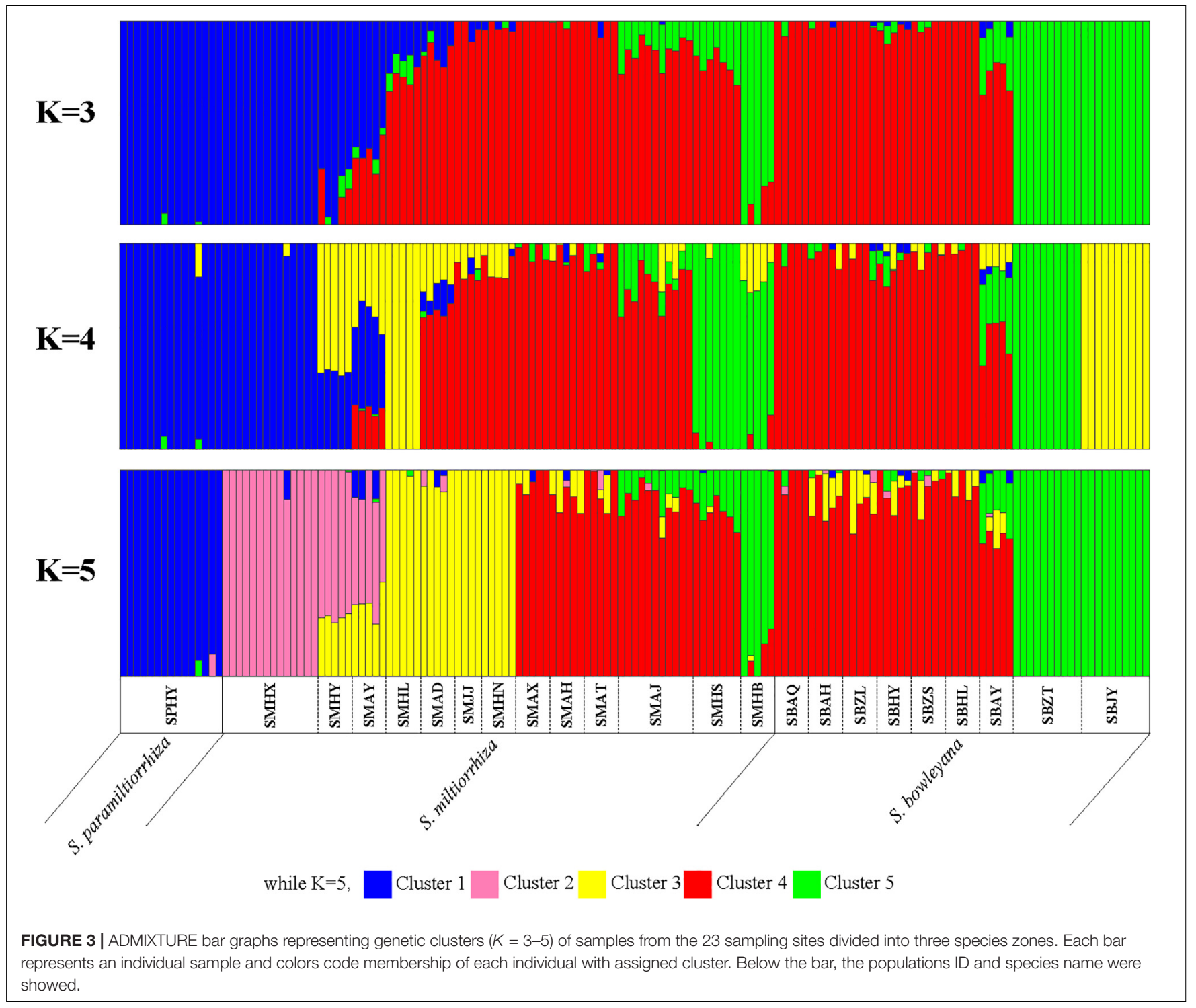

contributed to the mixture between these two species. The species used in this study were carefully identified, nonetheless, one could actually be hybrids with morphological similarity to one of the parent species. Although unidirectional migration of gene flow is detected from S. bowleyana to S. miltiorrhiza in BAYESASS analyses, we observed reciprocal admixtures between populations of these two species (Figure 3), which is also indicated by the low inter-specific differentiation $(0.1312)$ by AMOVA (Table 2). The weakly supported subclades in clade C might indicates the influence of partially contradict informative SNPs introduced by the gene flow between populations of these two species. Moreover, S. miltiorrhiza and S. bowleyana blossom at the same time and share pollinators (field observation), which indicate that the reproductive barriers between them might be weak to some extent, leading to inter-specific gene flow. On the other hand, since these two species occur in partial allopatry, the undifferentiated genetic divergence could also be a result of retention of ancestral polymorphism, as incomplete lineage sorting could lead to a similar phenomenon which was observed in many other closely related taxa (Alexander et al., 2017; Goetze et al., 2017; Cerca et al., 2021). Pairwise $F_{s t}$ estimates between SMAX, SMAH, SMAT, SMHB from S. miltiorrhiza and population SBHL, SBHY, SBZS, SBZL, SBAH, SBAQ of S. bowleyana were not statistically significant (Supplementary Table 2). In phylogenetic analysis, clade B \& C, dominated by populations from cluster 4 , is sister to clade $\mathrm{D}$, which is dominated by populations from cluster 5. Nonetheless, PCA analysis suggested a closer distance of the mixed cluster 4 and cluster 3 (S. miltiorrhiza populations in clade A). This indicated that both species could potentially contributed to the ancestry of cluster 4. It is also noteworthy that populations of S. miltiorrhiza (SMHB, SMHS, SMAJ) and S. bowleyana (SBZT, SBJY, SBAY) in cluster 5 did not mixed to each other as showed in PCA plot (Figure 4), despite sharing the same genetic ancestry revealed by Admixture analysis. Geographically, population SMHB is distantly located to SBZT/SBJY, indicating low recent gene flow 

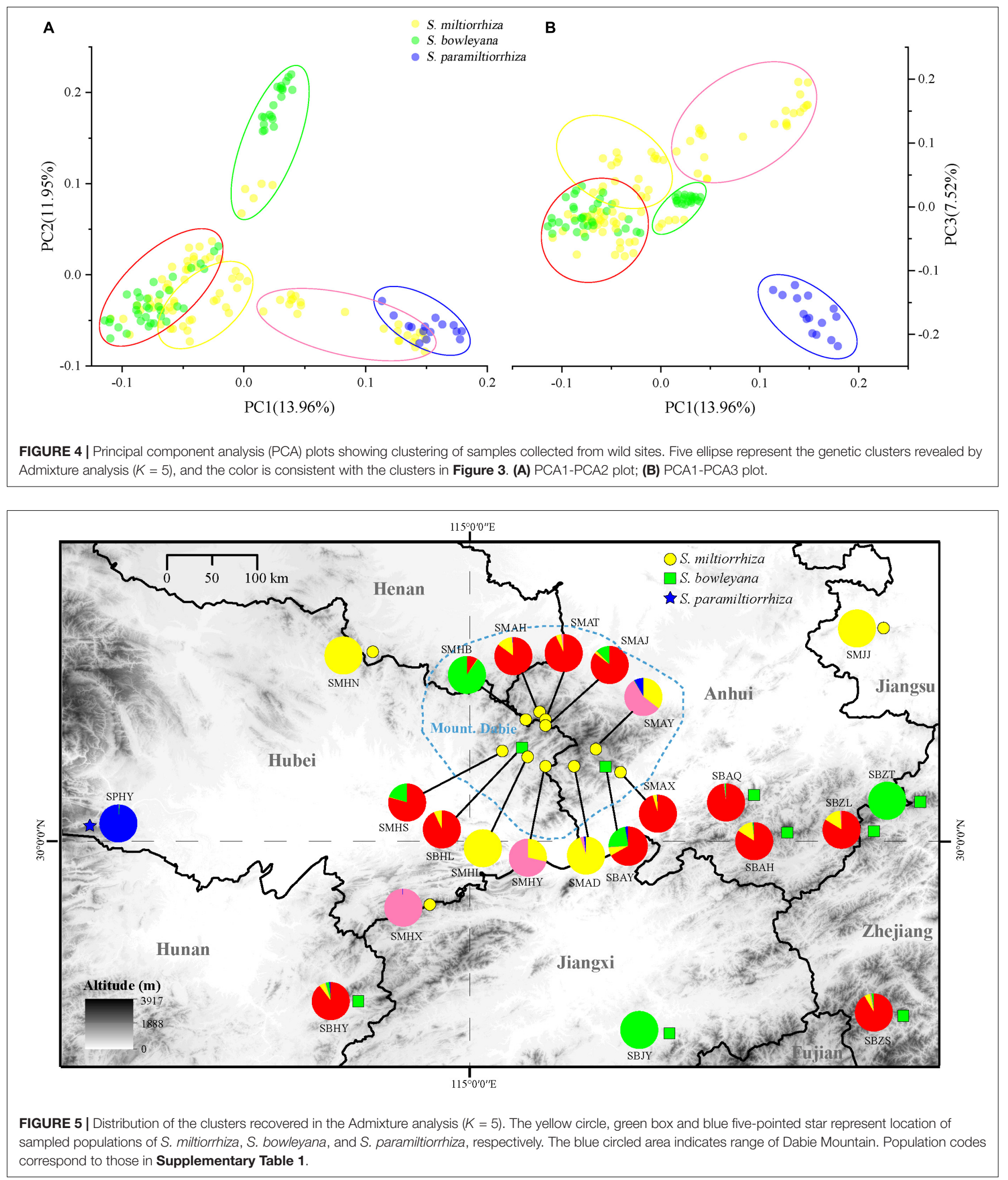

may occur between them. It suggests a retention of ancestral polymorphism of cluster 5 in both species. The AMOVA revealed different genetic differentiation scenarios of S. miltiorrhiza and
S. bowleyana. Differentiation among populations within clusters of S. bowleyana is high, and composed most of the genetic variation (56.36\%). However, in S. miltiorrhiza, most of variation 
TABLE 2 | Analysis of molecular variance (AMOVA) showing genetic variation among and within three studied species from collection sites.

\begin{tabular}{|c|c|c|c|c|c|}
\hline $\begin{array}{l}\text { Source of } \\
\text { variation }\end{array}$ & Sum Sq & $\begin{array}{c}\text { Variance } \\
\text { components }\end{array}$ & $\begin{array}{c}\% \text { of } \\
\text { variation }\end{array}$ & $P$-value & F-Statistics \\
\hline \multicolumn{6}{|c|}{ Interspecific comparison } \\
\hline $\begin{array}{l}\text { Among three } \\
\text { species }\end{array}$ & 108340.00 & 704.14 & 13.12 & 0.076 & 0.1312 \\
\hline $\begin{array}{l}\text { Among } \\
\text { populations } \\
\text { within species }\end{array}$ & 295574.20 & 1989.97 & 37.09 & 0.001 & 0.4270 \\
\hline $\begin{array}{l}\text { Within } \\
\text { populations }\end{array}$ & 341878.20 & 2670.92 & 49.78 & 0.001 & 0.5022 \\
\hline \multicolumn{6}{|c|}{ Salvia miltiorrhiza } \\
\hline Among Clusters & 52783.73 & 566.43 & 15.35 & 0.001 & 0.1535 \\
\hline $\begin{array}{l}\text { Among } \\
\text { populations } \\
\text { within Clusters }\end{array}$ & 62582.16 & 706.21 & 19.13 & 0.001 & 0.2260 \\
\hline $\begin{array}{l}\text { Within } \\
\text { populations }\end{array}$ & 162008.66 & 2418.04 & 65.52 & 0.001 & 0.3448 \\
\hline \multicolumn{6}{|c|}{ Salvia bowleyana } \\
\hline Among Clusters & 74212.78 & 1346.46 & 16.46 & 0.197 & 0.1646 \\
\hline $\begin{array}{l}\text { Among } \\
\text { populations } \\
\text { within Clusters }\end{array}$ & 199950.89 & 4609.83 & 56.36 & 0.001 & 0.6747 \\
\hline $\begin{array}{l}\text { Within } \\
\text { populations }\end{array}$ & 102235.60 & 2222.51 & 27.17 & 0.001 & 0.7283 \\
\hline
\end{tabular}

were detected within populations, and low genetic differentiation were observed in all group settings. This might imply that in spite of frequent gene follow, these two species might experienced differential evolutionary history. Further studies on S. miltiorrhiza and S. bowleyana at a larger population scale will be necessary to clarify the boundary between these two species.

Our analyses provided insights into the distribution of genetic diversity in S. miltiorrhiza, S. bowleyana, and S. paramiltiorrhiza, revealing that populations located in the Dabie Mountain range harbor the greatest amount of diversity (Figure 5). This area could potentially serve as a connection between $S$. miltiorrhiza from the north and $S$. bowleyana from the south. They might have experienced continuous gene flow over time, increasing toward the present day. According to the demographic history of S. miltiorrhiza and S. bowleyana illustrated by stairway plot, they experienced a contemporary bottleneck occurred at 8-6 kya (Figure 6), which was consistent with the $8.2 \mathrm{k}$ event of global cooling and population reduction in the Holocene epoch (Alley and Agustsdottir, 2005; Wicks and Mithen, 2014), which imply that S. miltiorrhiza and $S$. bowleyana may have shared refugia during climatic oscillations, where hybridization occurred. Salvia miltiorrhiza experienced an additional population contraction occurred at ca. 60 kya, which was consistent with the abrupt climate change and ecological turnover in late Pleistocene glaciation period (Finlayson and Carrión, 2007; Astakhov, 2008). Salvia bowleyana and S. paramiltiorrhiza might avoid the contraction during the glaciation due to their further south distribution range compare to $S$. miltiorrhiza. Therefore, genetic exchange could also occur among them when S. miltiorrhiza retreated
TABLE 3 | Posterior means of contemporary migration rates among species and genetic clusters estimated by BAYESASS (values in brackets are standard deviations, and mean values more than 0.1 are considered significant).

\begin{tabular}{|c|c|}
\hline Populations & Migration rates \\
\hline $\mathrm{SB} \rightarrow \mathrm{SM}$ & $\mathbf{0 . 2 1 4 5}(0.0219)$ \\
\hline $\mathrm{SB} \rightarrow \mathrm{SP}$ & 0.0060 (0.0058) \\
\hline $\mathrm{SM} \rightarrow \mathrm{SB}$ & $0.0156(0.0076)$ \\
\hline $\mathrm{SM} \rightarrow \mathrm{SP}$ & $0.0040(0.0040)$ \\
\hline $\mathrm{SP} \rightarrow \mathrm{SB}$ & $0.0175(0.0165)$ \\
\hline $\mathrm{SP} \rightarrow \mathrm{SM}$ & $0.0250(0.0217)$ \\
\hline cluster $1 \rightarrow$ cluster 4 & $0.0158(0.0152)$ \\
\hline cluster $1 \rightarrow$ cluster2 & $0.0323(0.0211)$ \\
\hline cluster $1 \rightarrow$ cluster 3 & $0.0165(0.0157)$ \\
\hline cluster $1 \rightarrow$ cluster 5 & $0.0158(0.0152)$ \\
\hline cluster2 $\rightarrow$ cluster 4 & $0.0227(0.0152)$ \\
\hline cluster $2 \rightarrow$ cluster 3 & $0.0115(0.0110)$ \\
\hline cluster2 $\rightarrow$ cluster 5 & $0.0115(0.0113)$ \\
\hline cluster $2 \rightarrow$ cluster 1 & $0.0118(0.0115)$ \\
\hline cluster3 $\rightarrow$ cluster 4 & $0.0256(0.0175)$ \\
\hline cluster3 $\rightarrow$ cluster2 & $0.0134(0.0131)$ \\
\hline cluster $3 \rightarrow$ cluster 5 & $0.0134(0.0127)$ \\
\hline cluster $3 \rightarrow$ cluster 1 & $0.0134(0.0128)$ \\
\hline cluster4 $\rightarrow$ cluster2 & $0.0046(0.0045)$ \\
\hline cluster4 $\rightarrow$ cluster 3 & $0.0191(0.0092)$ \\
\hline cluster $4 \rightarrow$ cluster 5 & $0.0238(0.0101)$ \\
\hline cluster4 $\rightarrow$ cluster 1 & $0.0048(0.0047)$ \\
\hline cluster5 $\rightarrow$ cluster 4 & $0.0644(0.0235)$ \\
\hline cluster5 $\rightarrow$ cluster2 & 0.0109 (0.0105) \\
\hline cluster5 $\rightarrow$ cluster3 & 0.0106 (0.0105) \\
\hline cluster $5 \rightarrow$ cluster 1 & $0.0107(0.0102)$ \\
\hline
\end{tabular}

SM: S. miltiorrhiza; SB: S. bowleyana; SP: S. paramiltiorrhiza. The bold value indicates strong signal of contemporary migration.

further south during that period. Population expansion of both S. miltiorrhiza and S. bowleyana took place during 6-4 kya, after which the population size of both species reached a stable status to present. No recent reduction in population size was observed based on genetic data. However, due to recent excessive excavation and habitat destruction, the wild populations of S. miltiorrhiza and its relatives reduced drastically. This could be explained by that the populations we sampled in the study were mostly collected in the nature reserves, where human activities are rare and restricted. Moreover, the population destruction started just decades ago, as such the role of genetic drift will be limited over such a short period of time (Johnson et al., 2018). Otherwise, the frequent gene flow among the species could also contributed to their maintenance of genetic diversity.

Our study suggests a complex scenario of inter/intra-specific evolutionary history of among S. miltiorrhiza, S. bowleyana, and $S$. paramiltiorrhiza. Incomplete lineage sorting, repeated gene flow, as well as localized events of hybridization together might have shaped the current genetic structure and intermixed relationship among these three closely related species. The molecular data indicates that $S$. paramiltiorrhiza exhibits a unique lineage after comparing with S. miltiorrhiza and S. bowleyana populations. Additionally, several novel compounds has been 


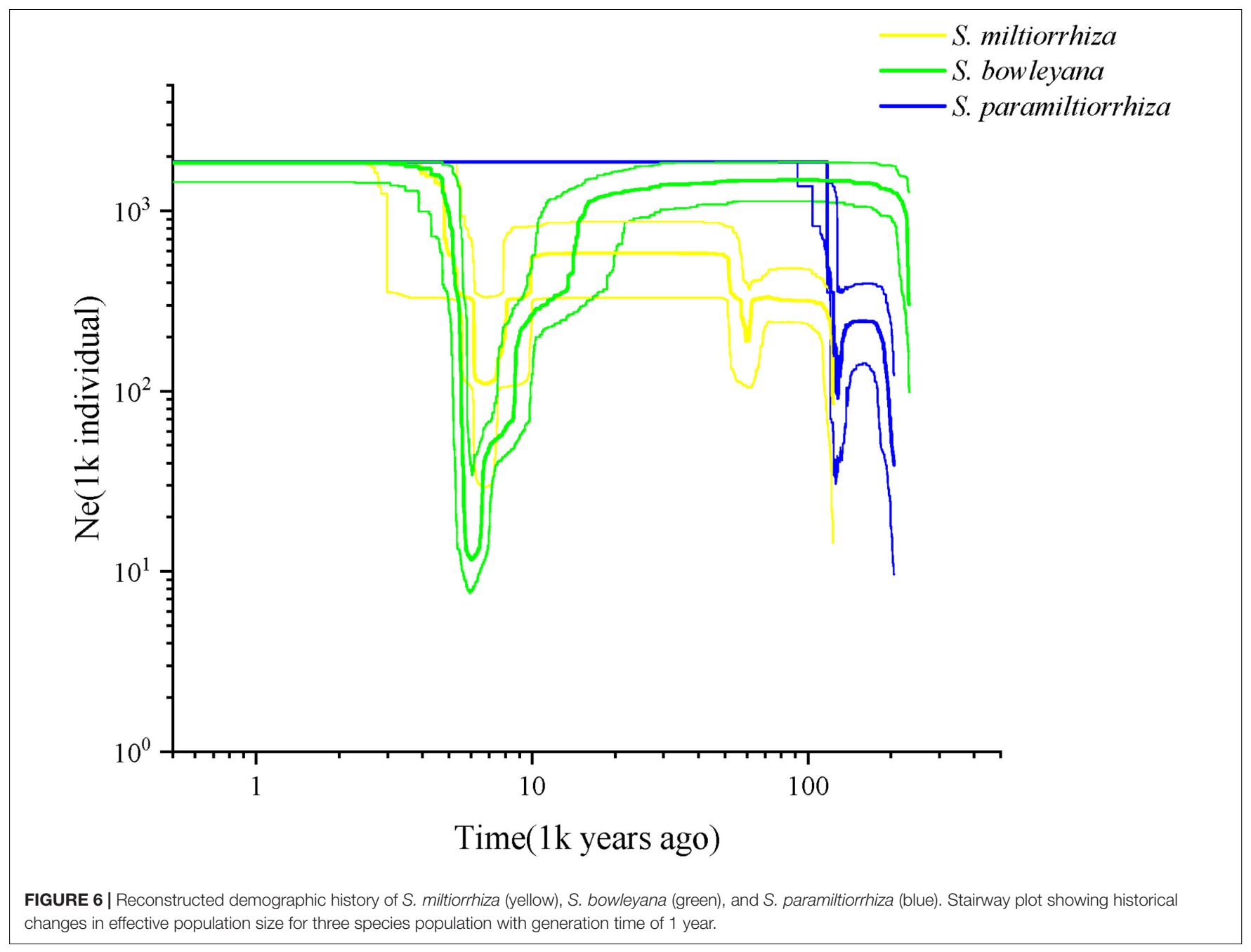

discovered from the roots of S. paramiltiorrhiza (Sun et al., 1991, 1992; Lin and Chang, 2000). For S. bowleyana, a complex interspecific interaction history between $S$. miltiorrhiza was revealed. It leads to a better understanding of the evolutionary history and dynamics of this species complex group. As relatives with S. miltiorrhiza, S. paramiltiorrhiza and S. bowleyana could serve as valuable wild germplasm resources to improve the quality of S. miltiorrhiza or develop novel breedings. Protection of the wild relatives of the targeted plant species will greatly enhance the scale of the genetic diversity and increase resilience to the agricultural system through breeding practice (Ford-Lloyd et al., 2011; Tyack et al., 2020). Therefore, we suggest that wild populations of S. paramiltiorrhiza and S. bowleyana should be considered together as whole with $S$. miltiorrhiza in wild germplasm conservation practice. For S. paramiltiorrhiza, on the basis of its restricted geographical distribution and threatened condition, we suggested that in situ conservation efforts should be made such as establishing protected areas and population recovering in natural habitat. Our study also revealed Dabie Mountain as a genetic diversity center and corridor of these three species. This region should be prioritized as a potential area of conservation, which would ensure the survival and maintain the evolutionary potential of the group. In the meantime, seed bank construction and ex situ conservation through botanical gardens are also recommended, as it also effectively preserve the biological and genetic diversity of S. miltiorrhiza, S. bowleyana and S. paramiltiorrhiza for sustainable usage. We hope this study would provide a guidance to the conservation of other medicinal plants when species history are complex and wild relatives are involved.

\section{DATA AVAILABILITY STATEMENT}

The raw sequencing data has been successfully uploaded to SRA in NCBI, https://www.ncbi.nlm.nih.gov/Traces/study/?acc= PRJNA716793.

\section{AUTHOR CONTRIBUTIONS}

Y-KW and Z-CQ conceived and designed the experiments and revised the manuscript. $\mathrm{Y}-\mathrm{BH}$ and $\mathrm{H}-\mathrm{WX}$ carried out 
investigation and collected materials. XZ, Z-CZ, J-JW, Z-CQ, and $\mathrm{Y}-\mathrm{KW}$ analyzed the data and wrote the manuscript. Y-KW and $\mathrm{Z}-\mathrm{CQ}$ acquired the funding. All authors contributed to the article and approved the submitted version.

\section{FUNDING}

This work was supported by the Natural Science Foundation of Zhejiang Province (LY21C030008), the Specific Project for Strategic Biological Resources and Technology Supporting System from the Chinese Academy of Sciences (ZSZY-001), the Science and Technology Program of Shanghai Science and Technology Committee (20392000600), the Chenshan Special Foundations from Shanghai Municipal Administration of Forestation and City Appearances (G102404 and F132423), Open Fund of Shanghai Key Laboratory of Plant Functional Genomics and Resources (PFGR202002 and PFGR202104), and the Open

\section{REFERENCES}

Alexander, A. M., Su, Y., Oliveros, C. H., Olson, K. V., Travers, S. L., and Brown, R. M. (2017). Genomic data reveals potential for hybridization, introgression, and incomplete lineage sorting to confound phylogenetic relationships in an adaptive radiation of narrow-mouth frogs. Evolution 71, 475-488. doi: 10.2307/ 48575966

Alexander, D. H., Novembre, J., and Lange, K. (2009). Fast model-based estimation of ancestry in unrelated individuals. Genome Res. 19, 1655-1664. doi: 10.1101/ gr.094052.109

Allen, G. C., Flores-Vergara, M. A., Krasynanski, S., Kumar, S., and Thompson, W. F. (2006). A modified protocol for rapid DNA isolation from plant tissues using cetyltrimethylammonium bromide. Nat. Protoc. 1, 2320-2325. doi: 10. 1038/nprot.2006.384

Alley, R., and Agustsdottir, A. (2005). The 8k event: cause and consequences of a major Holocene abrupt climate change. Q. Sci. Rev. 24, 1123-1149. doi: 10.1016/j.quascirev.2004.12.004

Andrews, K. R., Good, J. M., Miller, M. R., Luikart, G., and Hohenlohe, P. A. (2016). Harnessing the power of RADseq for ecological and evolutionary genomics. Nat. Rev. Genet. 17, 81-92. doi: 10.1038/nrg.2015.28

Astakhov, V. (2008). Geographical extremes in the glacial history of northern Eurasia: post-QUEEN considerations. Polar Res. 27, 280-288. doi: 10.1111/j. 1751-8369.2008.00069.x

Bentley, R. (2010). Medicinal plants. London: Domville-Fife Press.

Boczkowska, M., Bączek, K., Kosakowska, O., Rucińska, A., Podyma, W., and Wêglarz, Z. (2020). Genome-wide diversity analysis of Valeriana officinalis L. using DArT-seq derived SNP markers. Agronomy 10:1346. doi: 10.3390/ agronomy10091346

Bolger, A. M., Lohse, M., and Usadel, B. (2014). Trimmomatic: a flexible trimmer for Illumina sequence data. Bioinformatics 30, 2114-2120. doi: 10. 1093/bioinformatics/btu170

Canter, P. H., Thomas, H., and Ernst, E. (2005). Bringing medicinal plants into cultivation: opportunities and challenges for biotechnology. Trends Biotechnol. 23, 180-185. doi: 10.1016/j.tibtech.2005.02.002

Cerca, J., Rivera-Colón, A. G., Ferreira, M. S., Ravinet, M., Nowak, M. D., Catchen, J. M., et al. (2021). Incomplete lineage sorting and ancient admixture, and speciation without morphological change in ghost-worm cryptic species. PeerJ. 9:e10896. doi: 10.7717/peerj.10896

Chen, S., Yu, H., Luo, H., Wu, Q., Li, C., and Steinmetz, A. (2016). Conservation and sustainable use of medicinal plants: problems, progress, and prospects. Chin. Med. 11:37. doi: 10.1186/s13020-016-0108-7

Danecek, P., Auton, A., Abecasis, G., Albers, C. A., Banks, E., DePristo, M. A., et al. (2011). The variant call format and VCFtools. Bioinformatics 27, 2156-2158. doi: 10.1093/bioinformatics/btr330
Fund of Shaoxing Academy of Biomedicine of Zhejiang Sci-Tech University (SXAB202020).

\section{ACKNOWLEDGMENTS}

We would like to thank Gui-Bin $\mathrm{Li}$ for help with data analysis. We also thank the editor and two reviewers for their insightful comments and suggestions on the early version of the manuscript.

\section{SUPPLEMENTARY MATERIAL}

The Supplementary Material for this article can be found online at: https://www.frontiersin.org/articles/10.3389/fgene. 2021.688323/full\#supplementary-material

Davey, J. W., Hohenlohe, P. A., Etter, P. D., Boone, J. Q., Catchen, J. M., and Blaxter, M. L. (2011). Genome-wide genetic marker discovery and genotyping using next-generation sequencing. Nat. Rev. Genet. 12, 499-510. doi: 10.1038/ $\operatorname{nrg} 3012$

Doebley, J. F., Gaut, B. S., and Smith, B. D. (2006). The Molecular genetics of crop domestication. Cell. 127, 1309-1321. doi: 10.1016/j.cell.2006.12.006

Finlayson, C., and Carrión, J. S. (2007). Rapid ecological turnover and its impact on Neanderthal and other human populations. Trends Ecol. Evol. 22, 213-222. doi: 10.1016/j.tree.2007.02.001

Ford-Lloyd, B. V., Schmidt, M., Armstrong, S. J., Barazani, O., Engels, J., Hadas, R., et al. (2011). Crop Wild Relatives-Undervalued, Underutilized and under Threat? BioScience 61, 559-565. doi: 10.1525/bio.2011.61.7.10

Fu, X., Dou, J., Mao, J., Su, H., Jiao, W., Zhang, L., et al. (2013). RADtyping: an integrated package for accurate de novo codominant and dominant RAD genotyping in mapping populations. PLoS One 8:e79960. doi: 10.1371/journal. pone.0079960

Goetze, M., Zanella, C. M., Palma-Silva, C., Büttow, M. V., and Bered, F. (2017). Incomplete lineage sorting and hybridization in the evolutionary history of closely related, endemic yellow-flowered Aechmea species of subgenus Ortgiesia (Bromeliaceae). Am. J. Bot. 104, 1073-1087. doi: 10.3732/ajb.170 0103

Govindaraj, M., Vetriventhan, M., and Srinivasan, M. (2015). Importance of genetic diversity assessment in crop plants and its recent advances: An overview of its analytical perspectives. Genet. Res. Int. 2015, 1-14. doi: 10.1155/2015/ 431487

Guindon, S., Dufayard, J., Lefort, V., Anisimova, M., Hordijk, W., and Gascuel, O. (2010). New algorithms and methods to estimate maximum-likelihood phylogenies: Assessing the performance of PhyML 3.0. Syst. Biol. 59, 307-321. doi: $10.1093 /$ sysbio/syq010

Hamilton, A. C. (2004). Medicinal plants, conservation and livelihoods. Biodiv. Conserv. 13, 1477-1517. doi: 10.1023/B:BIOC.0000021333.23413.42

Hamilton, A. C. (2008). Medicinal plants in conservation and development: case studies and lessons learnt. Salisbury, UK: Plantlife International.

Hao, D. C., Gu, X., and Xiao, P. G. (2015). "14 - Phytochemical and biological research of Salvia medicinal resources," in Medicinal Plants (587-639), eds D. C. Hao, X. Gu, and P. G. Xiao (Sawston: Woodhead Publishing).

Heywood, V. H., and Iriondo, J. M. (2003). Plant conservation: old problems, new perspectives. Biol. Conserv. 113, 321-335. doi: 10.1016/S0006-3207(03) 00121-6

Hu, G., Takano, A., Drew, B. T., Liu, E., Soltis, D. E., Soltis, P. S., et al. (2018). Phylogeny and staminal evolution of Salvia (Lamiaceae, Nepetoideae) in East Asia. Ann. Bot. 2018:5045153. doi: 10.1093/aob/mcy104/5045153

Huang, X., and Li, H. (1981). Notes on Salvia Linn. in China. Acta Phytotaxonom. Sin. 19, 245-249. 
Jamshidi-Kia, F., Lorigooini, Z., and Amini-Khoei, H. (2018). Medicinal plants: Past history and future perspective. J. Herbmed. Pharm. 7, 1-7. doi: 10.15171/ jhp.2018.01

Johnson, M. T. J., Prashad, C. M., Lavoignat, M., and Saini, H. S. (2018). Contrasting the effects of natural selection, genetic drift and gene flow on urban evolution in white clover (Trifolium repens). Proc. R. Soc. B:Biol. Sci. 285:20181019. doi: 10.1098/rspb.2018.1019

Jombart, T., and Ahmed, I. (2011). adegenet 1.3-1: new tools for the analysis of genome-wide SNP data. Bioinformatics 27, 3070-3071. doi: 10.1093/ bioinformatics/btr521

Kalyaanamoorthy, S., Minh, B. Q., Wong, T. K., von Haeseler, A., and Jermiin, L. S. (2017). ModelFinder: fast model selection for accurate phylogenetic estimates. Nat. Methods 14:587. doi: 10.1038/nmeth.4285

Kamvar, Z. N., Brooks, J. C., and Grünwald, N. J. (2015). Novel R tools for analysis of genome-wide population genetic data with emphasis on clonality. Front. Genet. 6:208. doi: 10.3389/fgene.2015.00208

Kum, K. Y. (2019). Herbal products' quality control using 1H-NMR metabolomics approach - An example from Danshen (Salvia miltiorrhiza Bunge). Ph.D, London: University College London.

Lee, K. J., Lee, J., Sebastin, R., Cho, G., and Hyun, D. Y. (2020). Molecular genetic diversity and population structure of ginseng germplasm in RDA-Genebank: Implications for breeding and conservation. Agronomy 10:68. doi: 10.3390/ agronomy10010068

Lee, S., Choi, J., Lee, B., Yu, J., and Lim, C. E. (2018). Genetic diversity and structure of an endangered medicinal herb: implications for conservation. AoB Plants 10:y21. doi: 10.1093/aobpla/ply021

Li, Q., Li, M., Yuan, Q., Cui, Z., Huang, L., and Xiao, P. (2013). Phylogenetic relationships of Salvia (Lamiaceae) in China: Evidence from DNA sequence datasets. J. Syst. Evol. 51, 184-195. doi: 10.1111/j.1759-6831.2012. 00232.x

Li, R., Yu, C., Li, Y., Lam, T., Yiu, S., Kristiansen, K., et al. (2009). SOAP2: an improved ultrafast tool for short read alignment. Bioinformatics 25, 1966-1967. doi: 10.1093/bioinformatics/btp336

Lin, H., and Chang, W. (2000). Diterpenoids from Salvia miltiorrhiza. Phytochemistry 53, 951-953. doi: 10.1016/S0031-9422(99)00433-1

Liu, D., Zhang, L., Wang, J., and Ma, Y. (2020). Conservation genomics of a threatened Rhododendron: Contrasting patterns of population structure revealed from neutral and selected SNPs. Front. Genet. 11:00757. doi: 10.3389/ fgene.2020.00757

Liu, X., and Fu, Y. (2015). Exploring population size changes using SNP frequency spectra. Nat. Genet. 47, 555-559. doi: 10.1038/ng.3254

Minh, B. Q., Nguyen, M. A. T., and von Haeseler, A. (2013). Ultrafast Approximation for Phylogenetic Bootstrap. Mol. Biol. Evol. 30, 1188-1195. doi: 10.1093/molbev/mst024

Minh, B. Q., Schmidt, H. A., Chernomor, O., Schrempf, D., Woodhams, M. D., von Haeseler, A., et al. (2020). IQ-TREE 2: New Models and Efficient Methods for Phylogenetic Inference in the Genomic Era. Mol. Biol. Evol. 37, 1530-1534. doi: 10.1093/molbev/msaa015

Peakall, R., and Smouse, P. E. (2012). GenAlEx 6.5: genetic analysis in Excel. Population genetic software for teaching and research-an update. Bioinformatics 28, 2537-2539. doi: 10.1093/bioinformatics/bts460

Peterson, B. K., Weber, J. N., Kay, E. H., Fisher, H. S., and Hoekstra, H. E. (2012). Double digest RADseq: An inexpensive method for de novo SNP discovery and genotyping in model and non-model species. PLoS One. 7:e37135. doi: 10.1371/journal.pone.0037135

Purcell, S., Neale, B., Todd-Brown, K., Thomas, L., Ferreira, M. A. R., Bender, D., et al. (2007). PLINK: a tool set for whole-genome association and populationbased linkage analyses. Am. J. Hum. Genet. 81, 559-575. doi: 10.1086/519795

R Development Core Team (2018). R: A Language and Environment for Statistical Computing. Vienna: R Foundation for Statistical Computing.

Rambaut, A., Drummond, A. J., Xie, D., Baele, G., and Suchard, M. A. (2018). Posterior summarisation in Bayesian phylogenetics using Tracer 1.7. Syst. Biol. 67, 901-904.

Rochette, N. C., Rivera Colón, A. G., and Catchen, J. M. (2019). Stacks 2: Analytical methods for paired-end sequencing improve RADseqbased population genomics. Mol. Ecol. 28, 4737-4754. doi: 10.1111/mec. 15253
Sarwat, M., Nabi, G., Das, S., and Srivastava, P. S. (2011). Molecular markers in medicinal plant biotechnology: past and present. Crit. Rev. Biotechnol. 32, 74-92. doi: 10.3109/07388551.2011.551872

Schippmann, U., Leaman, D., and Cunningham, A. (2002). Impact of Cultivation and Gathering of Medicinal Plants on Biodiversity: Global Trends and Issues Biodiversity and the ecosystem approach in agriculture, forestry and fisheries (142-167). Rome: Inter-Departmental Working Group on Biological Diversity for Food and Agriculture.

Schippmann, U., Leaman, D., and Cunningham, A. (2006). A comparison of cultivation and wild collection of medicinal and aromatic plants under sustainability aspects. Med. Aromat. Plants 17:6. doi: 10.1007/1-4020-5449-1_6

Sun, X., Luo, H., and Sakai, T. (1992). Pentacyclic Triterpenes from Salvia paramittiorrhiza. Japan. Soc. Pharm. 46, 202-205.

Sun, X., Luo, H., Sakai, T., and Niwa, M. (1991). Paramiltioic acid, a novel rearranged norabietanoid from Salvia paramiltiorrhiza. Tetrahedron Lett. 32, 5797-5800. doi: 10.1016/S0040-4039(00)93558-5

Tyack, N., Dempewolf, H., and Khoury, C. K. (2020). The potential of payment for ecosystem services for crop wild relative conservation. Plants 9:101305. doi: $10.3390 /$ plants9101305

Vining, K. J., Hummer, K. E., Bassil, N. V., Lange, B. M., Khoury, C. K., and Carver, D. (2020). Crop wild relatives as germplasm resource for cultivar improvement in mint (Mentha L.). Front. Plant Sci. 11:01217. doi: 10.3389/fpls.2020.01217

Wang, B. (2010). Salvia miltiorrhiza: Chemical and pharmacological review of a medicinal plant. J. Med. Plants Res. 4, 2813-2820. doi: 10.1590/S0102695X2011005000024

Wang, S., Liu, P., Lv, J., Li, Y., Cheng, T., Zhang, L., et al. (2016). Serial sequencing of isolength RAD tags for cost-efficient genome-wide profiling of genetic and epigenetic variations. Nat. Protoc. 11, 2189-2200. doi: 10.1038/nprot.2016.133

Wang, S., Meyer, E., McKay, J. K., and Matz, M. V. (2012). 2b-RAD: a simple and flexible method for genome-wide genotyping. Nat. Methods 9, 808-810. doi: 10.1038/nmeth.2023

Wang, X., Liu, X., Ko, Y., Jin, X., Sun, J., Zhao, Z., et al. (2020). Genetic diversity and phylogeography of the important medical herb, cultivated Huang-Lian populations, and the wild relatives Coptis species in China. Front. Genet. 11:00708. doi: 10.3389/fgene.2020.00708

WHO, IUCN, and WWF (1993). Guidelines on the Conservation of Medicinal Plants. Gland: International Union for Conservation of Nature and Natural Resources.

Wicks, K., and Mithen, S. (2014). The impact of the abrupt 8.2 ka cold event on the Mesolithic population of western Scotland: a Bayesian chronological analysis using 'activity events' as a population proxy. J. Archaeol. Sci. 45, 240-269. doi: $10.1016 /$ j.jas.2014.02.003

Wilson, G. A., and Rannala, B. (2003). Bayesian inference of recent migration rates using multilocus genotypes. Genetics 163, 1177-1191. doi: 10.1093/eurpub/ 13.1.11

Xu, H., Song, J., Luo, H., Zhang, Y., and Li, Q. (2016). Analysis of the genome sequence of the medicinal plant Salvia miltiorrhiza. Mol. Plant. 6, 949-952. doi: 10.1016/j.molp.2016.03.010

Yang, A., Wu, B., Shen, C., Zhang, G., Liu, H., Guo, H., et al. (2017). Microsatellite records for volume 9, issue 3. Conserv. Genet. Resour. 9, 507-511. doi: 10.1007/ s12686-017-0806-1

Zhang, Y., Yan, Y., Wu, Y., Hua, W., Chen, C., Ge, Q., et al. (2014). Pathway engineering for phenolic acid accumulations in Salvia miltiorrhiza by combinational genetic manipulation. Metab. Eng. 21, 71-80. doi: 10.1016/ j.ymben.2013.10.009

Conflict of Interest: The authors declare that the research was conducted in the absence of any commercial or financial relationships that could be construed as a potential conflict of interest.

Copyright (C) 2021 Zhou, Zhang, Huang, Xiao, Wu, Qi and Wei. This is an openaccess article distributed under the terms of the Creative Commons Attribution License (CC BY). The use, distribution or reproduction in other forums is permitted, provided the original author(s) and the copyright owner(s) are credited and that the original publication in this journal is cited, in accordance with accepted academic practice. No use, distribution or reproduction is permitted which does not comply with these terms. 